\title{
IAMJ
}

INTERNATIONAL AYURVEDIC MEDICAL JOURNAL

\section{A REVIEW ON THERAPEUTIC EFFECT OF DHAVADI KWATH IN THE MANAGEMENT OF INDRALUPTA}

\author{
Manjari Chandravanshi ${ }^{1}$, Balendra Singh $^{2}$, Uttam Nirmalkar $^{3}$, Satrupa Nirmal $^{4}$ \\ ${ }^{1}$ MS Scholar, P.G. Department of Shalya tantra, GAC Raipur C.G., India \\ ${ }^{2}$ Reader \& HOD, P.G. Department of Shalya tantra, GAC Raipur C.G., India \\ ${ }^{3}$ Lecturer, P.G. Department of Shalya tantra, GAC Raipur C.G., India \\ ${ }^{4}$ Lecturer, P.G. Department of Shalya tantra, GAC Raipur C.G., India \\ Corresponding Author: manjarikwd308@gmail.com
}

https://doi.org/10.46607/iamj1409022021

(Published online: February 2021)

Open Access

(C) International Ayurvedic Medical Journal, India 2021

Article Received: 12/01/2021 - Peer Reviewed: 28/01/2021 - Accepted for Publication: 07/02/2021

\section{Check for updates}

\begin{abstract}
Hair is an indication of both social and professional status. Hair fall is common in both male and female. Alopecia refers to sudden or gradual hair loss. According to symptoms Alopecia can be correlated with Indralupta. Indralupta is a disease which is mainly due to vitiation of Tridosha and Rakta Dhatu. Ayurveda propagates equal importance to curative and preventive aspects. Various medicated oil and Lepa for local application are available in Ayurvedic texts for the management of Indralupta. Acharya Harita have mentioned Dhavadi Kwath for Shiroprakshalana (Head bath) in Indralupta Chikitsa. It acts as Kapha-Pitta Shamaka and Raktasodhaka with Kashaya, Tikta and Madhura rasa \& sheet veerya properties. It clears the obstruction of Romakupa and improves the circulation of blood on the scalp. Dhavadi Kwath tackle microorganism that causes infections by own antibacterial and antifungal properties.
\end{abstract}

Keywords: Indralupta, Alopecia, Dhavadi Kwath.

\section{INTRODUCTION}

Hair is considered as essential and protective part of the body. Hair fall and hair thinning is therefore a sensitive issue for both sexes of all races. Faulty dietary habits, lifestyle, stressful living, in appropriate nutrition, infection, allergic manifestation, use of medication, traumatic, surgeries, auto-immune disease etc. 
can triggers hairfall ${ }^{1}$. Increased hair fall in Ayurveda is termed as Indralupta, Khalitya and Rujya ${ }^{2}$. According to Acharya Sushruta Pitta along with Vata by involving root of hairs (Romakupa) making to fall off and thereafter Kapha together with Rakta obstructs the channels of Romakupa leading to the stoppage of the growth of new hairs. This condition is known as Indralupta $^{2}$. Indralupta is due to over intake of Kshara ${ }^{3}$, Lavana $^{4}$,Viruddha Ahara ${ }^{3}$, and use of Ushna-Tikshna Ahara. All these increased the Pitta Dosha and Affects the Rakta Dhatu. Indralupta can be correlated with Alopecia in modern. Alopecia is a condition characterised by hair loss from the scalp ${ }^{5}$. Ayurveda offers different effective treatment for Indralupta like Shiroabhayanga, Lepa, Rasayan, Nasya, Shiravedhana etc $^{6}$. Acharya Harita has mention the Dhavadi Kwath for Shiroprakshalana ${ }^{7}$ (head bath). Dhavadi Kwath contains having Kapha-Pitta Shamaka, Sheeta Veerya, Raktsodhaka, and Vishaghna properties. It helps in cleanses the channels, opens the pores which were blocked and enhance the blood circulation over the affected area and promoting growth of the new hairs.

Table1: Drug description: following ingredients all used for the preparations of Dhavadi Kwath-

\begin{tabular}{|l|l|l|l|l|}
\hline S. No & Drug Name & Botanical Name & Useful Part & Praporation \\
\hline 1. & Dhava & Anogeissus latifolia & Bark & 1 Part \\
\hline 2. & Arjuna & Terminalia arjuna & Bark & 1 Part \\
\hline 3. & Kadamb & Anthocephalus chinensis & Bark & 1 Part \\
\hline 4. & Shirish & Albizia lebbeck & Bark & 1 Part \\
\hline 5. & Rohitaka & Tecoma undulata & Bark & 1 Part \\
\hline
\end{tabular}

Table 2: Pharmacological and therapeutic Properties of all ingredients of the Dhavadi Kwath: -

\begin{tabular}{|c|c|c|c|c|c|c|c|}
\hline $\begin{array}{l}\text { Drug } \\
\text { Name }\end{array}$ & Rasa & Guna & Veerya & Vipaka & Doshghnata & karma & $\begin{array}{l}\text { Pharmacological } \\
\text { Properties }\end{array}$ \\
\hline Dhava ${ }^{8}$ & $\begin{array}{l}\text { Madhura, } \\
\text { Kashaya, } \\
\text { Katu. }\end{array}$ & $\begin{array}{l}\text { Laghu, } \\
\text { Ruksha. }\end{array}$ & Sheeta. & Katu. & $\begin{array}{l}\text { Kaphapitta } \\
\text { Shamaka. }\end{array}$ & $\begin{array}{l}\text { Rasayana, Vishaghna, } \\
\text { Vranaropana, Shotha- } \\
\text { hara }\end{array}$ & $\begin{array}{l}\text { CNS depressant } \\
\text { activity, }{ }^{9}\end{array}$ \\
\hline Arjuna $^{10}$ & Kashaya & $\begin{array}{l}\text { Laghu, } \\
\text { Ruksha }\end{array}$ & Sheeta & Katu & $\begin{array}{l}\text { Kaphapitta } \\
\text { Shamaka. }\end{array}$ & $\begin{array}{l}\text { Raktaprasadana, } \\
\text { Kaphaghna, Vishaghna, } \\
\text { Vranaropana, Shotha- } \\
\text { hara }\end{array}$ & $\begin{array}{l}\text { cytotoxic, antifun- } \\
\text { gal, antibacterial, }{ }^{11}\end{array}$ \\
\hline Kadamba $a^{12}$ & $\begin{array}{l}\text { Kashaya, } \\
\text { Madhura, } \\
\text { Lavana. }\end{array}$ & Ruksha & Sheeta & Katu & $\begin{array}{l}\text { Vatapitta } \\
\text { Shamaka. }\end{array}$ & $\begin{array}{l}\text { Amapachan, Deepana, } \\
\text { Vishaghna, Shothahara, } \\
\text { Varnya, Dhatuvardha- } \\
\text { ka. }\end{array}$ & $\begin{array}{l}\text { Anti-hepatotoxic, } \\
\text { antihelmintic, } \\
\text { antibacterial. } \\
\text { Antiinflammatry, }{ }^{13}\end{array}$ \\
\hline Shirisha ${ }^{14}$ & $\begin{array}{l}\text { Madhura, } \\
\text { Tikta, } \\
\text { Kashaya. }\end{array}$ & $\begin{array}{l}\text { Laghu, } \\
\text { Ruksha, } \\
\text { Teekshna. }\end{array}$ & Sheeta. & Katu & $\begin{array}{l}\text { Tridosha } \\
\text { Shamaka. }\end{array}$ & $\begin{array}{l}\text { Raktashodhaka, } \\
\text { Kaphaghna, Vishaghna, } \\
\text { Shothahara, Varnya, } \\
\text { Shirovirechana. }\end{array}$ & $\begin{array}{l}\text { Antiprotozoal, anti- } \\
\text { fungal, } \\
\text { anti-allergic, anal- } \\
\text { gesic, } \\
\text { antibacterial, CNS } \\
\text { depressant }^{15}\end{array}$ \\
\hline Rohitaka ${ }^{16}$ & $\begin{array}{l}\text { Katu, Tikta, } \\
\text { Kashaya. }\end{array}$ & $\begin{array}{l}\text { Laghu, } \\
\text { Ruksha }\end{array}$ & Sheeta & Katu & $\begin{array}{l}\text { Kaphapitta } \\
\text { Shamaka. }\end{array}$ & $\begin{array}{l}\text { Raktashodhaka, } \\
\text { Vishaghna, Lekhana, } \\
\text { Krimighna, Deepana }\end{array}$ & $\begin{array}{l}\text { Antibacterial, anti- } \\
\text { fungal, antiviral, } \\
\text { analgesic. } \\
\text { anticancer, CNS } \\
\text { depressant, }{ }^{17}\end{array}$ \\
\hline
\end{tabular}




\section{DISCUSSION}

Indralupta is disorder due to vitiation of all Doshas. However, Pitta vitiation is the predominant factor. Dhavadi Kwath subsides Pitta. Which pacifies the burning sensation and vitiated Rakta because all ingredient of Dhavadi Kwath are having Sheeta $V_{\text {eerya }}{ }^{18}$. Kapha block the hair follicles by not allowing fresh ones to grow. Most ingredients of the Dhavadi Kwath have Kashaya Rasa, Tikta Rasa, Madhura Rasa predominantly. Kashaya Rasa have Lekhana, Chhedana, Shodhana properties. it helps to absorbing Kleda and clears the Romakupa ${ }^{19}$ (hair follicles). Tikta Rasa acts as Amashoshaka, Srotoshodhaka and Kapha Chhedaka. Which removes Ama and toxins from hair roots. And opens the channels of the head and improves oxygenation ${ }^{20}$. Madhura Rasa is Keshya. It subsides Vata and Pitta and calms the central nervous system (Indriyaprasadhana) ${ }^{21}$. All contents of Dhavadi Kwath having Ruksha and Laghu Guna. Ruksha Guna absorbing Kleda which restrict the further block of Romakupa. ${ }^{22}$ Laghu Guna acts as Srotoshodhana. It reduces Kapha and Malas. ${ }^{23}$ Dhavadi Kwath having antifungal, antibacterial, antihelmintic, antiallergic properties. It helps in the treatment of infections (eczema, freckles and ringworm) and allergies. Dhavadi Kwath also acts as CNS depressant. It can slow brain activity, making them useful for treating stress. Ultimately it helps in improving scalp health and promote hair growth.

\section{CONCLUSION}

Dhavadi Kwath protects the hair against dandruff and Krimi that could cause hair loss. It helps in the strengthening hair shafts and bosting hair growth. Dhavadi Kwath also balances vitiated Doshas and cleanses channels witch helpful for nourishment of hairs. This drug can be effective for treatment of Indralupta.

\section{REFERENCES}

1. Golwalla's medicine, edited by ASPIF, Golwalla and Sharukh A. Golwalla, Edition- $23^{\text {th }}$, Published by Dr. Aspi F. Golwalla, Dermatology, 2011, P.N-772-774.
2. Sushruta Samhita translated by prof. G.D. Singhal and Colleagues. Publication by Chaukhambha Sanskrita pratisthana Delhi, edition-2 ${ }^{\text {nd }}, 2007$, Su. Ni 13/33-34, Voll-I, P.N-595.

3. Charaka Samhita of Agnivesha, edited with Charaka Chandrika, Hindi commentary by Dr. Brahmanand Tripathi publication, Chaukhamba Surbharati Prakashan Varanasi, Vol-I, edition 2013, Cha.Vi-1/18, P.N 661.

4. Charaka Samhita of Agnivesha, edited with Charaka Chandrika, Hindi commentary by Dr. Brahmanand Tripathi publication, Chaukhamba Surbharati Prakashan Varanasi, Vol-I, edition 2013, Cha.Vi-1/17, P.N 661.

5. API textbook of medicine, voll-1, $9^{\text {th }}$ edition-2012, Editor- In Chief Yash Pal Mujal, published by-The Association of physicians of India; Disorders of Skin Appendages Page No-509.

6. Sushruta Samhita translated by prof. G.D. Singhal and Colleagues. Publication by Chaukhambha Sanskrita pratisthana Delhi, edition-2 ${ }^{\text {nd }}, 2007$, Voll II, Su. Chi 20/24-26, P.N.348.

7. Harita Samhita, Editor Pt-Hariharprasad Tripathi, edition $2^{\text {nd }}, 2009$, published by Chaukhamba Sanskrita Academy, Varanasi, Chapter 43/7, P.N-433,

8. Dravya Guna vigyan edited by- P.V. Sharma, Published by Chaukhamba Orientalia, Varanasi, Print2007, Part-II, Page No-674-675.

9. Database on medicine plant used in Ayurveda Voll-I, P.C.Sharma, M.B.Yelne, T.J. Dennis, published by CCRAS, print- 2005, Page No-128-19.

10. Dravya Guna vigyan edited by- P.V. Sharma, Published by Chaukhamba Orientalia, Varanasi, Print2007, Part-II, Page No-195-196.

11. Database on medicine plant used in Ayurveda Voll-III, P.C.Sharma, M.B.Yelne, T.J. Dennis, published by CCRAS, print- 2004, Page No-58-59.

12. Dravya Guna vigyan edited by- P.V. Sharma, Published by Chaukhamba Orientalia, Varanasi, Print2007, Part-II, Page No-41-42.

13. Database on medicine plant used in Ayurveda Voll-IV, P.C.Sharma, M.B.Yelne, T.J. Dennis, published by CCRAS, print- 2002, Page No-243-244.

14. Dravya Guna vigyan edited by- P.V. Sharma, Published by Chaukhamba Orientalia, Varanasi, Print2007, Part-II, Page No-774.

15. Database on medicine plant used in Ayurveda Voll-I, P.C.Sharma, M.B.Yelne, T.J. Dennis, published by CCRAS, print- 2005, Page No-446-47. 
16. Dravya Guna vigyan edited by- P.V. Sharma, Published by Chaukhamba Orientalia, Varanasi, Print2007, Part-II, Page No-553.

17. Database on medicine plant used in Ayurveda Voll-VI, P.C.Sharma, M.B.Yelne, T.J. Dennis, published by CCRAS, print- 2004, Page No-322-23.

18. Dravya Guna vigyan edited by- J.L.N Sastry, Published by Chaukhamba Orientalia, Varanasi, Print-2016, PartI, Chapter 7/2, Page No-165.

19. Dravya Guna vigyan edited by- J.L.N Sastry, Published by Chaukhamba Orientalia, Varanasi, Print-2016, PartI, Chapter 5/6/1, Page No-117.

20. Dravya Guna vigyan edited by- J.L.N Sastry, Published by Chaukhamba Orientalia, Varanasi, Print-2016, PartI, Chapter 5/5/1, Page No-116.

21. Dravya Guna vigyan edited by- J.L.N Sastry, Published by Chaukhamba Orientalia, Varanasi, Print-2016, PartI, Chapter 5/1/2, Page No-114.

22. Dravya Guna vigyan edited by- J.L.N Sastry, Published by Chaukhamba Orientalia, Varanasi, Print-2016, PartI, Chapter 4/6, Page No- 57.

23. Dravya Guna vigyan edited by- J.L.N Sastry, Published by Chaukhamba Orientalia, Varanasi, Print-2016, PartI, Chapter 4/2, Page No-54.

\section{Source of Support: Nil \\ Conflict of Interest: None Declared}

How to cite this URL: Manjari Chandravanshi et al: A Review On Therapeutic Effect Of Dhavadi Kwath In The Management Of Indralupta. International Ayurvedic Medical Journal \{online\} 2021 \{cited February, 2021\} Available from: http://www.iamj.in/posts/images/upload/411_414.pdf 